\title{
Successful Management of Refractory Prosthetic Vessel Infection, Prosthetic Valve Endocarditis and Mediastinitis: Report of a Case-Late Timing Caused Repeated Surgery
}

\author{
Takashi Ando*, Daichi Akiyama, Hioshi Okada and Makoto Takeda \\ Department of Cardiovascular Surgery, Yokohama Rosai Byoin, Yokohama, Kanagawa, Japan
}

\begin{abstract}
The patient was diagnosed with anuloaortic ectasia with localized dissection in the right Valsalva sinus. He underwent aortic root replacement with mechanical valve. But, he was re-admitted for mediastinitis. Although his serum (CRP) level was normalized for one month, his body temperature suddenly rose to $40^{\circ} \mathrm{C}$. An emergency operation was performed including re-sternotomy, drainage, and irrigation. However, a proximal anastomotic site ruptured and he went into shock. Following cardiopulmonary bypass, we performed a second aortic root replacement. Therefore, the patient was operated for sternum debridement and to wrap the prosthetic vessel into an omental pedicle. Nine days after the last intervention, bleeding from the chest wound suddenly appeared and he went into shock. He was transported to the operating room to initiate cardiopulmonary bypass. During circulatory arrest, laceration was detected at the same annular position. Deep into the left ventricle, we made interrupted sutures in the left cardiac muscle. Next, we implanted a Freestyle aortic root bioprosthesis using the full root technique. After removing all former implants, another prosthetic vessel was anastomosed between the Freestyle conduit and the distal aorta, and wrapped with omental pedicle. After three weeks, his serum CRP level was normal. He remained free of infection for at least three years.
\end{abstract}

Keywords: Prosthetic vessel infection; Freestyle aortic root bioprosthesis; Prosthetic valve endocarditis; Mediastinitis; Omental pedicle

Abbreviations: CRP: C-Reactive Protein; CT: Computed Tomography; MSSA: Methicillin-Sensitive Staphylococcus aureus; PVE: Prosthetic Valve Endocarditis

\section{Introduction}

We report a case of life-threatening cardiac surgery complication including mediastinitis, graft infection, and anastomotic failure after aortic root replacement. It is essential to conduct aggressive debridement and to divert blood flow away from the infected tissue. The patient was successfully treated by thorough debridement, followed by aortic root replacement with a Freestyle stentless bioprosthesis and prosthetic vessel wrapped in an omental pedicle.

\section{Case Report}

A 42-year-old man visited our hospital in March 2014 complaining of shortness of breath. Based on an echocardiography and computed tomography, he was diagnosed with anuloaortic ectasia, (maximum diameter, $85 \mathrm{~mm}$ ), with localized dissection in the right Valsalva sinus. His medical history included diabetes mellitus controlled with oral antidiabetic drugs for the past two years. In March 2014, he underwent aortic root replacement (operation 1) with a mechanical valve, $(25 \mathrm{~mm}$ Regent series, St. Jude Medical, MN, US) and a prosthetic vessel, (28 mm Triplex, Terumo Co., Tokyo, Japan). He was discharged without complication.

However, 20 days postoperatively, in April 2014, he developed a high fever after median sternotomy leading to sternum instability. Therefore, he was re-admitted urgently to our hospital for mediastinitis treatment including chest drainage therapy for pus buildup. His body temperature was $38.6^{\circ} \mathrm{C}$, pulse was regular, heart rate 90 beats $/ \mathrm{min}$, and blood pressure 102/70 mmHg. Abnormal laboratory findings included high levels of procalcitonin $(0.22 \mathrm{ng} / \mathrm{mL}$; normal range: $<0.01 \mathrm{ng} / \mathrm{mL})$ and CRP $(27.88 \mathrm{mg} / \mathrm{dL}$; normal range: $<0.2 \mathrm{mg} / \mathrm{dL})$, and high leukocyte count $(18800 / \mathrm{mL}$; normal range: $4,500-10,000 / \mathrm{mL})$. Echocardiography revealed hyperkinetic movement without prosthetic valve endocarditis. CT showed air effusion below the sternum and subcutaneous dead space. MSSA was isolated from the wound culture, but the blood culture was free of pathogens.

The patient was treated by intravenous administration of sensitive antibiotics (cefazolin $6 \mathrm{~g}$ /day), hyperbaric oxygen therapy, and daily debridement using a vacuum-assisted closure device. Intravenous cefazolin was continued until his serum CRP level was normalized. A follow-up CT showed that the air effusion below the sternum had disappeared, but the subcutaneous dead space remained.

Two weeks post-admission, he became afebrile. One month post-admission, his serum CRP level was normalized. Therefore, the cefazolin was terminated. However, 2 days post-cefazolin, he developed a gout attack, and a transient fever. Then 4 days post antibiotics, his body temperature suddenly rose to $40^{\circ} \mathrm{C}$, with chills and rigor. Various culture specimens were collected to identify the pathogen responsible for these symptoms of infection. At that time, a CT showed no increase in air effusion below the sternum. Therefore, we re-started the Cefazolin treatment. However, his laboratory data did not improve

*Corresponding author: Takashi Ando, MD, PhD, Department of Cardiovascular Surgery, Yokohama Rosai Byoin, Yokohama, Kanagawa, Japan, Tel: +81454748111; Fax: 81454748323; E-mail: taka@marianna-u.ac.jp

Received August 02, 2017; Accepted August 18, 2017; Published August 24 2017

Citation: Ando T, Akiyama D, Okada H, Takeda M (2017) Successful Management of Refractory Prosthetic Vessel Infection, Prosthetic Valve Endocarditis and Mediastinitis: Report of a Case-Late Timing Caused Repeated Surgery. J Vasc Med Surg 5: 328. doi: 10.4172/2329-6925.1000328

Copyright: ( 2017 Ando T, et al. This is an open-access article distributed unde the terms of the Creative Commons Attribution License, which permits unrestricted use, distribution, and reproduction in any medium, provided the original author and source are credited. 
Citation: Ando T, Akiyama D, Okada H, Takeda M (2017) Successful Management of Refractory Prosthetic Vessel Infection, Prosthetic Valve Endocarditis and Mediastinitis: Report of a Case-Late Timing Caused Repeated Surgery. J Vasc Med Surg 5: 328. doi: 10.4172/23296925.1000328

Page 2 of 3

over $48 \mathrm{~h}$ : (procalcitonin, $5.86 \mathrm{ng} / \mathrm{mL}$; CRP, $27.91 \mathrm{mg} / \mathrm{dL}$; leukocyte count, 10,500/mL). Two days later, MSSA was isolated from the blood cultures. Enhanced CT detected a large abscess around the artificial blood vessel, though the air below the sternum had disappeared (Figure 1). However, an echocardiography showed no mechanical valve dysfunction or aortic annular abscess.

An emergency operation was performed including re-sternotomy, drainage, and irrigation (operation 2). However, a proximal anastomotic site ruptured and he went into shock. We promptly established a right femoral artery, (though very small diameter)femoral vein cardiopulmonary bypass. We excised the right coronary button, exposing the bleeding source as a huge annular abscess around the commissure of the left-right cusp. These tissues were very weak, but the debridement area was made as large as possible. Following thorough irrigation, we could not close annular abscess by xenopericardium sheet due to lack of tissues which endured securing the sheet. So we had to use the same prosthetic vessel, made interrupted sutures used 3-0 Black metalworking needle (Nespylen, Alfresa pharma Co., Osaka Japan). We reconstructed the right coronary button as the same fashion (operation 2). After operation, the patient's chest remained open. The chest tube was maintained in place for continuous irrigation and drainage. Two days later, the irrigation fluid was fair. Therefore, the patient was operated for sternum debridement and to wrap the prosthetic vessel into an omental pedicle to prevent from further infection. So the patient's chest had closed (operation 3). The mediastinal culture was negative for infection and the symptoms improved. However, he developed a compartment syndrome in the right leg because of stenosis after purse string suture for the cannulation.

Nine days after the last intervention, bleeding from the chest wound suddenly appeared and he went into shock. He was transported to the operating room to initiate cardiopulmonary bypass via the left external iliac artery (operation 4), kept the omental pedicle off the packing site and put the omentum, which wrapped in the moisture saline gauze, on the abdominal wall during operation. Then his body temperature was decreased to $20^{\circ} \mathrm{C}$. During circulatory arrest, laceration was detected at the same annular position. Deep into the left ventricle, we made interrupted sutures used 3-0 Regular metalworking needle (Nespylen, Alfresa pharma Co., Osaka, Japan) in the left cardiac muscle. Next, we implanted a Freestyle aortic root bioprosthesis $(25$ $\mathrm{mm}$ in diameter; Medtronic Inc., Minneapolis, MN, USA) using the full root technique (Figure 2). After removing all former implants, another prosthetic vessel, $(24 \times 10 \mathrm{~mm}$, Triplex, Terumo Co., Tokyo, Japan), was anastomosed between the Freestyle conduit and the distal

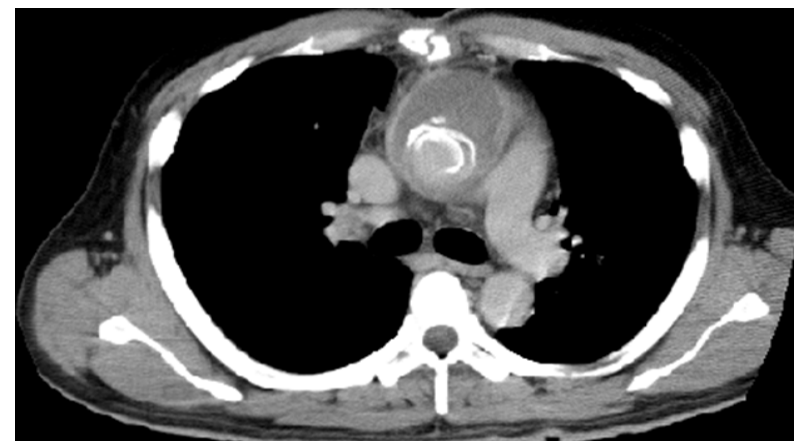

Figure 1: Computed tomography showed that the air effusion below the sternum was reduced by antibiotic therapy. However, a large effusion with enhanced capsule developed around the prosthetic vessel.
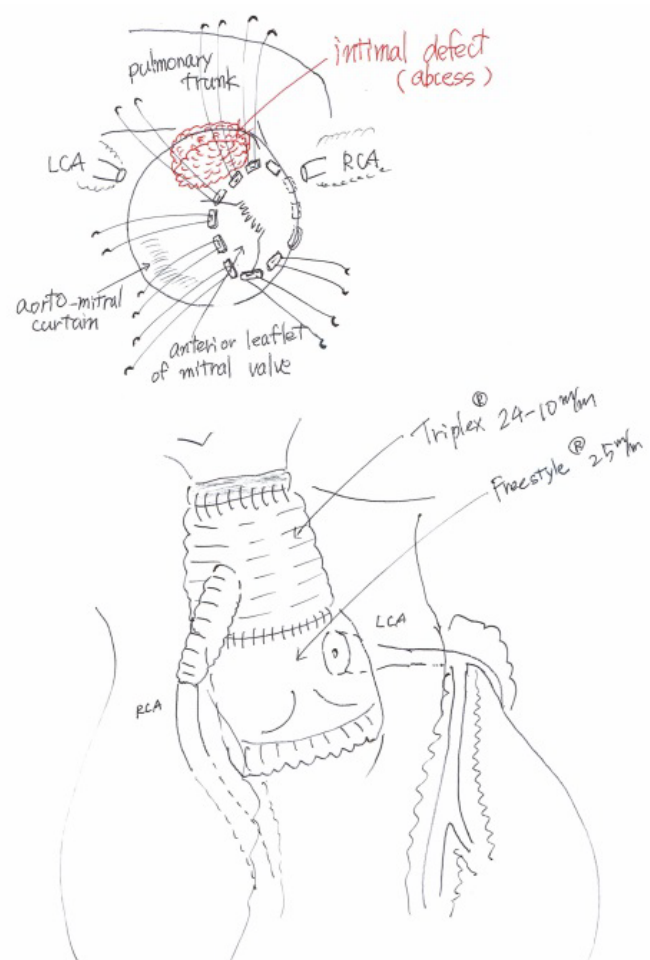

Figure 2: Schema of the last operation was shown. From surgeon's view, Step 1: Interrupted sutures were made deep into the muscle of the left ventricle. All medical implants were removed, including the left and right coronary buttons. Step 2: A Freestyle aortic root bioprosthesis was implanted by the full root technique. A new prosthetic vessel $(24 \times 10 \mathrm{~mm})$ was anastomosed between the bioprosthesis and distal aorta, using the branch for right coronary artery (RCA) reconstruction by the Piehler's method.

aorta, and re-wrapped with omental pedicle. Incidentally, MSSA was detected in cultures from the excised prosthetic vessel.

After the last aortic root replacement, the patient received both intravenous cefazolin and hyperbaric oxygen therapy. After three weeks, his serum CRP level was normal. He received intravenous Cefazolin during another three weeks, and then was shifted to oral antibiotics.

On day 110 post-admission, the patient was finally discharged after achieving independent gait with a walking harness. He has been visiting the outpatient clinic every month, and remained free of infection for at least three years.

\section{Discussion}

Heart surgery complications have become relatively rare, but those associated with infections have a high mortality rate, such as mediastinitis, prosthetic vessel infection, and PVE. Cases of annular abscess with PVE should receive an aortic homograft for the following reasons: hemodynamic optimizations, less prosthetic-patient mismatch, antithrombotic and resistance to infection [1]. But they seldom used by surgeons because they are difficult to acquire. Muller et al. treated 10 patients with the Freestyle aortic root bioprosthesis and concluded that it was an acceptable alternative for the treatment of severe endocarditis [2]. El-Hamamasy et al. reported that homograft roots exhibit significantly higher calcium scores than Freestyle roots 
Citation: Ando T, Akiyama D, Okada H, Takeda M (2017) Successful Management of Refractory Prosthetic Vessel Infection, Prosthetic Valve Endocarditis and Mediastinitis: Report of a Case-Late Timing Caused Repeated Surgery. J Vasc Med Surg 5: 328. doi: 10.4172/23296925.1000328

Page 3 of 3

due to faster early calcification [3]. Accordingly, there are many reports of PVE surgery using a Freestyle stentless bioprosthesis [4,5].

In our case, the last operation was too late. We suspected that the inflammation around the sternum and the prosthetic implants were due to bacteremia. After the one-month antibiotics treatment, a few days were sufficient for the bacterial infection to surge again. On the other hand, we had to use prosthesis with a mechanical valve and a prosthetic vessel in such an emergency situation. Even an omental pedicle wrap would not have been sufficient to prevent tissue infection, as shown by the MSSA positive cultures from the prosthetic vessel. Since we did not use a homograft, it was essential to conduct aggressive debridement and to divert blood flow away from the infected tissue. There is currently no information on the durability of xenogenic stentless valves (i.e., Freestyle stentless bioprosthesis) in young patients because most valves are replaced in patients $>50$ years old [1]. Longterm follow-up studies are required to further our evaluation of reinfection and durability $[1,6]$.

\section{Conclusion}

This report gives two informative data. The first point is that late timing caused repeated surgery ( 2 and 3 operations). The second point describes a successful treatment protocol for mediastinitis, graft infection, and anastomotic failure following root replacement using a Freestyle aortic root bioprosthesis with omental pedicle wrapping, after thorough debridement.

\section{Contributors}

TA conceived, designed and drafted the manuscript and was the principal operating surgeon. TA and DA was consultant in charge of patient's care. DA and $\mathrm{HO}$ assisted in the operation and collected various imaging data. TA and MT revised the manuscript. All authors have read and approved the final manuscript.

\section{Acknowledgments}

The authors would like to thank Enago (www.enago.jp) for the English language review.

\section{Patient Consent}

The authors have obtained written and signed consent to publish the case report from the patient.

\section{References}

1. Kobayashi J (2011) Stentless aortic valve replacement: an update. Vascular Health and Risk Management 7: 345-351.

2. Muller LC, Chevtchik O, Bonatti JO, Muller S, Fille M, et al. (2003) Treatment of destructive aortic valve endocarditis with the Freestyle aortic root bioprosthesis. Ann Thorac Surg 75: 453-456.

3. El-Hamamsy I, Zaki M, Stevens LM, Lucy AC, Michael R, et al. (2009) Rate of progression and functional significance of aortic root calcification after homograft versus Freestyle aortic root replacement. Circulation 120: S269-S275.

4. Siniawski H, Lehmkuhl H, Weng Y, Pasic M, Yankah C, et al. (2003) Stentless aortic valves as an alternative to homografts for valve replacement in active infective endocarditis complicated by ring abscess. Ann Thorac Surg 75: 803-808.

5. Perrotta S and Lentini S (2010) In patients with severe active aortic valve endocarditis, is a stentless valve as good as the homograft? Interactive Cardiovasc Thorac Surg 11: 309-313.

6. Nakamura H, Yamaguchi H, Nakao T, Oshima Y, Tokunaga N, et al. (2011) Reoperation for mid-term failure of a Freestyle bioprosthesis using a full root technique. Ann Thorac Cardiovasc Surg 17: 287-289. 